\title{
A Summary of the Myanmar Clinical Practice Guidelines for the Management of Obesity
}

\author{
Tint Swe Latt, Ko Ko, Than Than Aye, Aye Thidar, Ei Ei Khin \\ on behalf of the Myanmar Society of Endocrinology and Metabolism (MSEM) \\ University of Medicine Yangon, Myanmar
}

\begin{abstract}
There is a significant prevalence of overweight and obesity in Myanmar. It is anticipated that this will impact on the morbidity associated with other lifestyle diseases such as hypertension, Type 2 diabetes and various types of cancer. Recognizing this public health concern, the Myanmar Society of Endocrinology and Metabolism (MSEM) organized a task force for the development of evidence based clinical practice guidelines on obesity.
\end{abstract}

Key words: obesity, clinical practice guidelines, Myanmar

\section{Introduction}

The objective of the Myanmar Clinical Practice Guideline for Obesity is to develop an evidence based guideline for healthcare providers and the public by using data relevant for Myanmar people.

Obesity can be defined simply as a disease in which excess body fat has accumulated to such an extent that health may be adversely affected. However, the amount of excess fat, its distribution within the body, and the associated health consequences vary considerably between obese individuals.

The distribution of fat induced by weight gain affects the risks associated with obesity, and the kinds of disease that develop as a result of obesity. Excess abdominal fat is as great a risk factor for disease as is excess body fat per se. "Android obesity" is a result of abdominal fat distribution which is associated with increased risk for obesity related diseases (particularly cardiovascular diseases). In contrast "gynoid" fat distribution, in which fat is more evenly and peripherally distributed around the body, is less serious.

In Myanmar, according to the WHO stepwise approach to NCD surveillance in Yangon in 2003-2004, the prevalence of overweight (Body Mass Index [BMI] 25.0 - 29.9) was $20.6 \%$ in males and $29.96 \%$ in females, while the prevalence of obesity (BMI $\geq 30.0$ ) was $4.77 \%$ in males and $10.35 \%$ in females. According to the national survey carried out in 2009, the prevalence of overweight was $17.74 \%$ in males and $30.27 \%$ in females while the prevalence of obesity was $4.27 \%$ in males and $8.37 \%$ in females. For both sexes, the prevalence of overweight and obesity was $25.38 \%$ and $6.8 \%$ respectively.

ISSN 0857-1074

Copyright (C) 2011 by the JAFES

Received September 20, 2011. Accepted October 5, 2011.
Among children and adolescents aged 10-19 years in Yangon $7.6 \%$ were obese at the age of $10-19$ years in both sexes. Out of these $8.7 \%$ were boys and $6.5 \%$ were girls.

The increased risk of overweight and obesity in Myanmar substantially raises the risk of morbidity for hypertension, type 2 diabetes, stroke, gallbladder disease, osteoarthritis, sleep apnoea and respiratory problems, and endometrial, breast, prostate, and colon cancers in the country.

As a contributor to many medical problems and preventable death, overweight and obesity pose a public health challenge in Myanmar today.

The task force for Clinical Practice Guidelines on Obesity was formed by the members of the Myanmar Society of Endocrinology and Metabolism (MSEM). Relevant literature search was done. Recommendations on expected caloric needs and macronutrients proportions for Myanmar were developed by using local data.

\section{Management of Obesity}

\section{Management of Adult Obesity}

Obesity management encompasses the following four key strategies:

- $\quad$ prevention of weight gain

- $\quad$ promotion of weight maintenance

- management of obesity comorbidities

- $\quad$ promotion of weight loss

\section{Diagnosis and Assessment}

Since Myanmar is one of the member countries of the WHO South East Asia Region (SEAR) and previous 
studies on risk factors for noncommunicable diseases have followed the WHO guidelines, the task force decided to adopt the WHO classification for obesity in this guideline.

\section{Body mass index (BMI)}

Body mass index (BMI) $\mathrm{wt}(\mathrm{kg}) / \mathrm{ht}\left(\mathrm{m}^{2}\right)$ is an internationally accepted measure of general adiposity in adults. BMI takes account of the expected differences in weights of adults of different heights.

\section{Waist Circumference}

Waist circumference is at least as good an indicator of total body fat as BMI and is also the best anthropometric predictor of visceral fat.

Table 1. Classification of adult underweight, overweight and obesity according to BMI.

\begin{tabular}{|c|c|c|}
\hline Classification & BMI & Risk of comorbidities \\
\hline Under weight & $<18.50$ & $\begin{array}{l}\text { Low (but risk of other clinical } \\
\text { problems increased) }\end{array}$ \\
\hline $\begin{array}{l}\text { Normal range } \\
\text { Overweight: }\end{array}$ & $\begin{array}{l}18.50-24.99 \\
\geq 25.00\end{array}$ & Average \\
\hline Pre-obese & $25.00-29.99$ & Increased \\
\hline Obese class I & $30.00-34.99$ & Moderate \\
\hline Obese class II & $35.00-39.99$ & Severe \\
\hline Obese class III & $\geq 40.00$ & Very severe \\
\hline
\end{tabular}

Table 2. Sex-specific waist circumference and risk of metabolic complications associated with obesity

\begin{tabular}{lcc}
\hline \multirow{2}{*}{ Risk of metabolic complications } & \multicolumn{2}{c}{ Waist circumference (cm) } \\
\cline { 2 - 3 } & Men & Women \\
\hline Increased & $\geq 94$ & $\geq 80$ \\
Substantially increased & $\geq 102$ & $\geq 88$ \\
\hline Adapted from the NHRMC, 2003. & \multicolumn{2}{c}{}
\end{tabular}

The World Health Organization (WHO) recommended that an individual's relative risk of type 2 diabetes and cardiovascular disease could be more accurately classified using both BMI and waist circumference

\begin{tabular}{|c|c|c|c|c|}
\hline \multirow[b]{2}{*}{ Classification } & \multirow[b]{2}{*}{$\begin{array}{c}\mathrm{BMI} \\
\left(\mathrm{kg} / \mathrm{m}^{2}\right)\end{array}$} & \multirow[b]{2}{*}{ Class } & \multicolumn{2}{|c|}{$\begin{array}{l}\text { Disease Risk * Relative to } \\
\text { Normal Weight and Waist } \\
\text { Circumference (WC) }\end{array}$} \\
\hline & & & $\begin{array}{c}\text { Men WC } \\
94-102 \mathrm{~cm} \\
\text { Women WC } \\
80-8 \mathrm{~cm}\end{array}$ & $\begin{array}{c}\text { Men WC >102 cm } \\
\text { Women } \\
W C>88 \mathrm{~cm}\end{array}$ \\
\hline Normal weight $^{\dagger}$ & $18.5-24.9$ & & - & - \\
\hline Overweight & 25.0 & & Increased & High \\
\hline \multicolumn{5}{|l|}{ Obese } \\
\hline Mild & $30.0-34.9$ & Class I & High & Very high \\
\hline Moderate & $35.0-39.9$ & Class II & Very high & Very high \\
\hline Extreme & $40.0+$ & Class III & $\begin{array}{l}\text { Extremely } \\
\text { high }\end{array}$ & Extremely high \\
\hline \multicolumn{5}{|c|}{$\begin{array}{l}\text { * Disease risk for type } 2 \text { diabetes, hypertension, and cardiovascular } \\
\text { disease } \\
{ }^{t} \text { Increased waist circumference can also be a marker for increased risk } \\
\text { even in persons of normal weight } \\
\text { Obesity management should be implemented with the following five key } \\
\text { steps: }\end{array}$} \\
\hline
\end{tabular}

\section{Dietary Measures}

Achieving and sustaining appropriate body weight across the lifespan is vital to maintaining good health and quality of life. Calorie balance over time is the key to weight management. Calorie balance refers to the relationship between calories consumed from foods and beverages and calories expended in normal body functions (i.e., metabolic processes) and true physical activity.

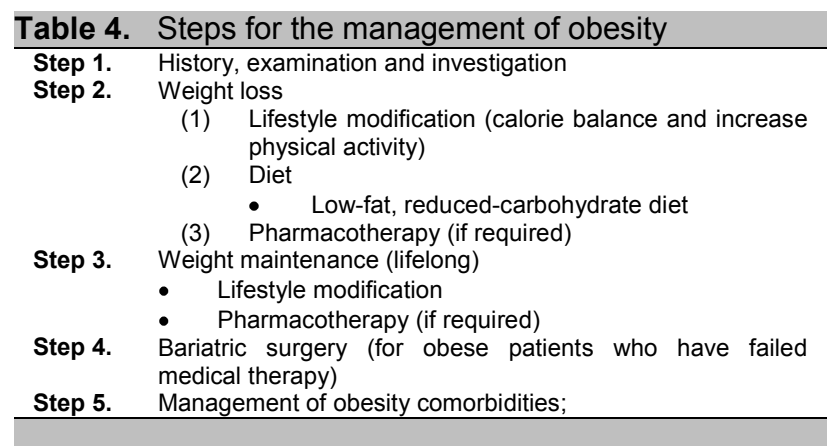

\begin{tabular}{ll} 
Table 5. Long - term goals for obesity therapy \\
\hline Criteria & Treatment success \\
\hline Reduction of excess weight & $7-10 \%$ of initial body weight \\
Maintenance of BMI & $<25 \mathrm{~kg} / \mathrm{m}^{2}$ \\
Blood pressure & any reduction \\
Blood glucose & any reduction \\
Glycaemic control $\left(\mathrm{HbA}_{1} \mathbf{c}\right)$ & any improvement \\
Other risk factors & any reduction \\
\hline
\end{tabular}

Calories consumed must equal calories expended for a person to maintain the same body weight. Consuming fewer calories than expended will result in weight loss, whereas, consuming more calories than expended will result in weight gain.

A calorie deficit of 500 calories or more per day should be an initial goal for weight loss for adults. A greater proportion of the calorie deficit should come from decreased caloric intake with a relatively smaller fraction from increased physical activity. The total number of calories a person needs each day varies depending on a number of factors, including the person's age, gender, height, weight, and level of physical activity. Table 6 provides estimated total caloric needs for weight maintenance based on age, gender and physical activity level for Myanmar.

Table 6. Estimated calorie needs per day by age, gender and physical activity level for Myanmar

\begin{tabular}{lcccc}
\hline Gender & $\begin{array}{c}\text { Age } \\
\text { (Years) }\end{array}$ & \multicolumn{3}{c}{ Physical Activity Level } \\
\cline { 3 - 5 } & & Sedentary & Active & $\begin{array}{c}\text { Moderately } \\
\text { Active }\end{array}$ \\
\hline Male & $15-18$ & $1700-2100$ & $2200-2600$ & $2600-3000$ \\
& $19-30$ & $2000-2400$ & $2400-2600$ & 2800 \\
& $31-60$ & $1800-2200$ & $2200-2400$ & $2600-2800$ \\
& $60+$ & $1600-1800$ & $2000-2200$ & $2200-2600$ \\
Female & $15-18$ & $1400-1600$ & $1800-2000$ & $2200-2400$ \\
& $19-30$ & $1600-1800$ & $1800-2000$ & $2000-2200$ \\
& $31-60$ & 1600 & 1800 & 2000 \\
& $60+$ & 1400 & 1600 & $1800-2000$ \\
\hline
\end{tabular}

To manage body weight, people should consume a diet that has an appropriate total number of calories and that is within the Acceptable Macronutrient Distribution Ranges (AMDR). Table 7 mentioned the AMDR which has taken into account both chronic disease risk reduction and intake of essential nutrients for the Myanmar population. 
Table 7. Recommended macronutrient proportions by age for Myanmar

\begin{tabular}{lccc}
\hline & Carbohydrates & Protein & Fat \\
\hline Young Children (1-3 yrs) & $45-65 \%$ & $5-20 \%$ & $30-40 \%$ \\
Older Children and & $45-65 \%$ & $10-30 \%$ & $25-35 \%$ \\
Adolescents (4-18 yrs) & & & \\
Adults (19 yrs and Older) & $55-65 \%$ & $10-15 \%$ & $15-30 \%$ \\
\hline
\end{tabular}

Carbohydrates, protein and fat should be the main sources of calories in the diet. In the Myanmar diet, most carbohydrates are consumed in the form of starches which are found in foods such as grains, potatoes and other starchy vegetables. A common source of starch in Myanmar diet is refined grains. Most of the Myanmar people tend to eat too much carbohydrates (in the form of rice), which constitutes more than $70 \%$ of the total caloric intake per day.

Myanmar cuisine usually contains too much fat in the form of cooking oil, which may constitute more than $35 \%$ of the total caloric intake per day. Traditionally, protein intake tends to be lower than $10 \%$ of the total calories per day.

It is recommended to consume 5 servings of vegetables and fruits every day. People should be cautious about consuming of sugar-sweetened beverages which provide excess calorie and few essential nutrients to the diet. They should only be consumed when nutrient needs have been met and without exceeding daily calorie limits. It is necessary to take into consideration calories from alcohol intake. Alcohol contributes 7 calories per gram. In the Yangon Division, $25.96 \%$ of men and $1.09 \%$ of women were current drinkers according to the 2003 - 2004 survey. The national survey on NCD risk factors in 2009 revealed that $31.17 \%$ of men and $1.47 \%$ of the women were current drinkers.

\section{Physical Activity}

Physical activity is the other side of the calorie balance equation and should be considered when addressing weight management. Table 8 shows the guidelines on the recommended physical activity for Myanmar which was adapted from the global guideline for physical activity for health.

\section{Behaviour therapy}

Individual or group-based psychological interventions should be included in weight management programmes. Psychological interventions should be tailored to the individual and their circumstances.

The range of appropriate psychological interventions and strategies includes:

- $\quad$ self monitoring of behaviour and progress

- $\quad$ stimulus control (where the patient is taught how to recognise and avoid triggers that prompt unplanned eating)

- cognitive restructuring (modifying unhelpful thoughts/thinking patterns)
- goal setting

- problem solving

- $\quad$ assertiveness training

- $\quad$ slowing the rate of eating

- reinforcement of changes

- relapse prevention

- $\quad$ strategies for dealing with weight regain

Table 8. Recommendation for physical activity Age Group (5 - 17 Years Old)

1. Children and youth aged $5-17$ years should accumulate at least 60 minutes of moderate - to vigorous-intensity physical activity daily.

2. Amounts of physical activity greater than 60 minutes provide additional health benefits.

3. Most of the daily physical activity should be aerobic Vigorous-intensity activities should be incorporated, including those that strengthen muscle and bone, at least 3 times per week.

Age Group (18 - 64 Years Old)

1) Adults aged $18-64$ should do at least 150 minutes of moderate-intensity aerobic physical activity throughout the week or do at least 75 minutes of vigorous-intensity aerobic physical activity throughout the week or an equivalent combination of moderate- and vigorous-intensity activity.

2) Aerobic activity should be performed in bouts of at least 10 minutes duration.

3) For additional health benefits, adults should increase their moderate-intensity aerobic physical activity to 300 minutes per week, or engage in 150 minutes of vigorous-intensity aerobic physical activity per week, or an equivalent combination of moderate-and vigorous-intensity activity.

4) Muscle-strengthening activities should be done involving major muscle groups on 2 or more days a week.

Age Group (65 Years Old and above)

1) Adults aged 65 years and above should do at least 150 minutes of moderate-intensity aerobic physical activity throughout the week or do at least 75 minutes of vigorousintensity aerobic physical activity throughout the week or an equivalent combination of moderate- and vigorous-intensity activity.

2) Aerobic activity should be performed in bouts of at least 10 minutes duration.

3) For additional health benefits, adults aged 65 years and above should increase their moderate-intensity aerobic physical activity to 300 minutes per week, or engage in 150 minutes of vigorous-intensity aerobic physical activity per week, or an equivalent combination of moderate-and vigorous-intensity activity.

4) Adults of this age group, with poor mobility, should perform physical activity to enhance balance and prevent falls on 3 or more days per week.

5) Muscle-strengthening activities should be done involving major muscle groups on 2 or more days a week.

6) When adults of this age group cannot do the recommended amounts of physical activity due to health conditions they should be as physically active as their abilities and conditions allow.

Adapted from the WHO Global Recommendations on Physical Activity for
Health. 2010.

\section{Pharmacotherapy}

Orlistat should be considered as an adjunct to lifestyle interventions in the management of obesity. Patients with BMI $\geq 25 \mathrm{~kg} / \mathrm{m}^{2}$ (with comorbidities) or BMI $\geq 30$ $\mathrm{kg} / \mathrm{m}^{2}$ should be considered on an individual case basis following assessment of risk and benefit.

Therapy with orlistat should be continued beyond 12 weeks only if the patient has lost at least $5 \%$ of their initial body weight from the start of drug treatment. Therapy should then be continued for as long as there are clinical benefits (e.g., prevention of significant weight regain).This may involve medication use outside current license. Ongoing risks and benefits should be discussed with patients. 


\section{Surgery}

Bariatric surgery should be considered on an individual case basis following assessment of risk/benefit in patients who fulfill the following criteria:

- Failure to respond to non-surgical treatment including dietary measures and weightreducing drugs.

- Individuals considering surgery should have received adequate information and understand and accept operative risks.

- Patients with a BMI $>40$, or BMI $>35$ with highrisk, life-threatening comorbid conditions.

Surgery should be undertaken only by an experienced surgeon in an appropriate clinical setting under expert medical surveillance, and with access to ventilator facilities and the support of a multidisciplinary team.

\section{Management of Risk Factors and Comorbidity}

The following risk factors and comorbidities should be assessed and managed accordingly:

Comorbidities:

- Coronary heart disease

- Other atherosclerotic disease

- Type 2 diabetes

- $\quad$ Sleep apnoea

- Other obesity associated diseases and

Risk factors:

- Smoking

- Hypertension

- High LDL-C

- Low HDL-C

- Impaired fasting glucose

- Family history of premature CHD

- More than 45 years (male) and more than 55 years (female)

\section{Overall Approach to Treatment}

\section{Management of Obesity in Children}

Obesity is used to refer to children and youth between the ages of 2 and 18 years who have BMI equal to or greater than the $95^{\text {th }}$ percentiles of the age and gender specific BMI charts developed by WHO.
In Pediatrics, the term "Obese" is not used to describe the degree of overweight. It is a general descriptive term, but does not necessarily indicate a certain level of overweight.

\section{Classification of Weight by BMI in Myanmar (in Children)}

Obesity is used to refer to children and youth between the ages of 2 and 18 years who have body mass indexes equal to or greater than the $95^{\text {th }}$ percentile of the age and gender specific BMI charts developed by WHO.

Overweight $-\mathrm{BMI}$ is $85^{\text {th }}-94^{\text {th }}$ percentile.

Obesity $-\mathrm{BMI}$ is $95^{\text {th }}-98^{\text {th }}$ percentile.

The overall goals for the family in management of overweight child and adolescence are:

- Strive to maintain current weight as the child grows.

- Consistently adhere to healthier life style patterns to prevent further weight gain.

- Encourage healthy lifestyle patterns for all families.

\section{Short-term goals}

- $10 \%$ loss of initial body weight in 6 months

- Physical activity of at least 1 hour per day

- Decreased television screen time to $<2$ hours per day

- Abstinence from sugar-sweetened beverages

- Intake of proper, balanced meals, including daily breakfast

\section{Long-term goals}

- Sustaining an altered lifestyle with behaviors that provide further weight loss or that maintain a declined weight, or that avoid additional weight gain; and maintaining a BMI < 85th percentile, although BMI between 85 th - 94th percentile may be healthy in some children

- Refer to a specialist for further assessment in the case of severe obesity where medical risks are present

- Drugs are not recommended for weight loss in children

- Weight loss surgery is not recommended in children

Table 9. Treatment options for different levels of BMI and other risk factors in Myanmar

\begin{tabular}{|c|c|c|c|c|c|}
\hline & Diet & Activity & Drug & VLCD & Surgery \\
\hline \multicolumn{6}{|l|}{ BMI $23-25 \mathrm{~kg} / \mathrm{m}^{2}$} \\
\hline No additional risk & $\sqrt{ }$ & $\checkmark$ & $x$ & & \\
\hline Increased WC & $\sqrt{ }$ & $\sqrt{ }$ & $\mathrm{x}$ & & \\
\hline $\mathrm{DM} / \mathrm{CHD} / \mathrm{HT} / \mathrm{HL}$ & $\sqrt{ }$ & $\sqrt{ }$ & $\sqrt{ }$ & & \\
\hline \multicolumn{6}{|l|}{ BMI $25-30 \mathrm{~kg} / \mathrm{m}^{2}$} \\
\hline No additional risk & $\checkmark$ & $\checkmark$ & $\sqrt{ }($ Consider $)$ & & \\
\hline Increased WC & $\sqrt{ }$ & $\sqrt{ }$ & $\sqrt{ }$ (Consider) & & \\
\hline $\mathrm{DM} / \mathrm{CHD} / \mathrm{HT} / \mathrm{HL}$ & $\sqrt{ }$ & $\sqrt{ }$ & $\sqrt{ }$ & & \\
\hline \multicolumn{6}{|l|}{$\mathrm{BMI}>30 \mathrm{~kg} / \mathrm{m}^{2}$} \\
\hline No additional risk & $\sqrt{ }$ & $\sqrt{ }$ & $\sqrt{ }($ Consider $)$ & $\sqrt{ }$ & $\sqrt{ }$ \\
\hline Increased WC & $\sqrt{ }$ & $\sqrt{ }$ & $\sqrt{ }$ & (consider in severely & (consider in severely \\
\hline $\mathrm{DM} / \mathrm{CHD} / \mathrm{HT} / \mathrm{HL}$ & $\sqrt{ }($ Intensive $)$ & $\sqrt{ }($ Intensive $)$ & $\sqrt{ }$ & obese) & obese) \\
\hline
\end{tabular}




\section{Assessment}

All patients - should be evaluated using the following tests:

- Blood pressure measurements by using appropriate size cuff obtained on 3 separate occasions and evaluated against Pediatric norms

- Fasting blood sugar level

- Fasting lipid panel

- Liver function panel (ALT and AST) to evaluate for steato-hepatitis

When the child is at the $85^{\text {th }}$ percentile, evaluation begins at 10 years of age, then every 2 years.

Selected patients may need to be referred for specialist care. The following tests may also be requested depending on any suspected or identified comorbid conditions or secondary causes of obesity:

- $\quad$ Free T4 and TSH, if hypothyroidism is suspected

- 24 hours urine free cortisol and creatinine if Cushing's syndrome is suspected

- "Modified" glucose challenge obtaining fasting glucose and insulin level and then 2 hours post prandial glucose level if insulin resistance is suspected.

- $\quad$ For BMI > 95th percentile and 10 years or older, the following tests should be done: non- fasting lipid, HbA1c, ALT should be done

- If there is a positive family history of an early cardiovascular event, obtain lipids beginning at 2 years of age to rule out genetic dyslipidemia.

\section{Prevention of Childhood and Adolescent Obesity}

- Counsel about physical activity, sedentary behavior, meal patterns and food choice at every well care visit in all pediatric patients and their patients.

- Both bottle and breast fed children can be overfed, Families should be counseled that babies do not need to finish every bottle. Skim milk can replace whole milk after age two.

- Food should not be offered as a reward by parents and care givers.

- $\quad$ Eat healthy family meals together 3 to 6 times per week with limited food portions.

- $\quad$ Limit sugar - sweetened beverage.

- Recommend at least 5 serving of fruits and vegetables a week. A serving is $1 / 2$ cup.

- Recommend a healthy breakfast everyday. Obese children are more likely to skip breakfast or to eat smaller breakfast than leaner children.

- Limit TV time to $<2$ hours per day and remove TV from the child's bed room.

- Encourage 60 minutes of physical activity and free play each day.

- Limit eating outside the home, especially fast food.

\section{Treatment failure}

If there is no success with basic lifestyle intervention, motivated families should be referred to a family-based program which incorporates nutrition, physical activity and behavioral and involves $>25$ hours of contact over a 6 month period.

\section{Referral}

Consult/refer if comorbidities persist or if no improvement after 6 months of structured lifestyle.

\section{Conclusion}

In conclusion, the following strategies will help reduce obesity in Myanmar:

(1) Changing the lifestyles of the citizens such as avoiding the consumption of Western diets which are high in refined carbohydrates, saturated fats and sugars, and increasing physical activity and avoiding sedentary lifestyles;

(2) Narrowing down the significant gaps in medical services by strengthening the competencie of medical professionals in obesity management; and

(3) Strengthening the community infrastructure and public health systems so as to reduce under-nutrition as well as obesity.

\section{References}

1. KoKoZaw, Tint SweLatt, et. al, The study of National and Regional levels of obesity in Myanmar. 2009.

2. Tint SweLatt, PhyuPhyuAung, TheingiThwin, KoKoZaw (2004). WHO STEPwise approach to NCD surveillance, Yangon Division, Myanmar (2003 - 2004).

3. MaungMaungThanHtike, Tint SweLatt, KoKoZaw Myanmar STEP survey 2009; WHO STEPS chronic disease risk factor surveillance. 2009

4. WHO, Geneva Obesity: Preventing and Managing the Global Epidemic. 2000.

5. WHO Global Recommendations on Physical Activity for Health 2010.

6. Dietary Guideline Advisory Committee, USDA Dietary Guidelines for Americans 2010. www.dietaryguidelines.gov. 2010.

7. August GP, Caprio S, Fennoy I, Freemark M, et al. Prevention and Treatment of Pediatric Obesity: Journal of Clinical Endocrinology \& Metabolism. 2008; 93 (12): 4576-4599.

8. Caveney E, Caveney BJ, Somaratne R, Turner R, Gourgiotis L Pharmaceutical interventions for obesity: A public health perspective. Diabetes, Obesity and Metabolism. 2011 13: 490-497.

9. Padwal RS, Majumdar SR Drug treatments for obesity: Orlistat, sibutramine, and rimonabant. Lancet. 2007;369: 71-77.

10. Santry HP, Gillen DL, Lauderdale DS Trends in bariatric surgical procedures.JAMA. 2005;294: 1909-1917.

11. SIGN guideline. Management of obesity: A national clinical guideline. 2010

12. WHO. Obesity: Preventing and managing the global epidemic. Report of a WHO consultation. WHO Technical Report Series 894 Geneva: World Health Organization, 2000.

13. WHO. Appropriate Body-Mass Index for Asian populations and its implications for policy and intervention strategies. The Lancet, 2004; 363:157-163.

14. National Health \& Medical Research Council (NHMRC). Clinical practice guidelines for the management of overweight and obesity in adults. 2003. 